\title{
MEDIUM-TERM CARBOHYDRATE TOLERANCE IMPROVES AND THEN DETERIORATES WITH ADVANCING PREGNANCY IN URBAN NIGERIAN WOMEN
}

\author{
OLORONDU J', EMUVEYAN EE, * OHWOVORIOLE AE' \\ Department of Medicine, \\ College of Medicine, University of Lagos, Lagos, Nigeria. \\ Department of Obstetrics and Gynaecology, \\ College of Medicine, University of Lagos, Lagos, Nigeria
}

*Corresponding Author

\begin{abstract}
Background: It has been reported that carbohydrate metabolism does not deteriorate in pregnancy in the African, an observation at variance with general teaching.

Objective: To determine the effect of pregnancy on medium term carbohydrate metabolism

Methods: Medium term carbohydrate metabolism was evaluated in pregnant and non-pregnant Nigerian women using changes in Glycoted Plasma Proteins (GPP) as a surrogate for medium-term carbohydrate metabolism. Study subjects included women in all three trimesters of pregnancy, a group of non-pregnant women with normal carbohydrate metabolism and another group of women with confirmed diabetes already on treatment but not pregnant. Fasting plasma glucose was determined by the glucose oxidase method while GPP was determined using a modification of the thiobarbituric method with correction for levels of total plasma proteins (TPP). Average values are presented as mean (SD) while relationship between variables were determined using Pearson product moment correlation coefficients. Significance of $p$ values of determinations is set at $p<0.05$.

Results: Women in the various pregnancy trimesters were similar in age and without family or personal history of diabetes mellitus. Fasting plasma glucose (FPG) levels of pregnant women in all trimesters were lower than those of non-pregnant women of similar age. GPP values in the first and second trimesters were lower than non-pregnant women and third trimester pregnant women. GPP was highest in the third trimester of pregnancy even being significantly higher than in the non-pregnant women.

Correlation between FPG and GPP was highest and significant in the third semester.

Conclusion: FPG levels are poor reflections of the carbohydrate tolerance in pregnancy. Carbohydrate metabolism is enhanced in the first trimester and thereafter deteriorates throughout pregnancy. When using GPP to assess carbohydrate metabolism in pregnancy, correction should be made for the level of total plasma proteins. Carbohydrate metabolism in the African deteriorates with advancing pregnancy.
\end{abstract}

Keywords: Pregnancy, Carbohydrate intolerance, Glycated plasma proteins, Fructosamine, Fasting plasma glucose, Nigeria.

\section{INTRODUCTION}

Controversy surrounds the overall effect of pregnancy on carbohydrate metabolism (1-3). However majority opinion favours the view that pregnancy as a diabetogenic factor associated with deterioration in carbohydrate metabolism, which progressively gets worse with advancing gestational age. In Africa, the results of studies about carbohydrate metabolism in pregnancy have been disparate with some studies reporting deterioration as in the other parts of the world reporting. Some reports indicate an improvement in carbohydrate metabolism with advancing gestation age (3-7). There is consensus that fasting plasma glucose (FPG) levels are lower in the pregnant state than in the non-pregnant state especially in the early pregnancy. The controversy rests more on the response to a glucose or carbohydrate challenge in the various phases of pregnancy. Traditional tests on which these conclusions have been made were based on oral glucose tolerance test (OGTT), intravenous glucose tolerance test or casual blood glucose estimation (6-9). OGTT, the gold standard of carbohydrate tolerance assessment has been shown to produce very erratic results in pregnancy as glucose absorption shows wide variations during pregnancy, making the results of OGTT poorly reproducible where changes are not gross. OGTT in the various trimesters has therefore not been particularly helpful in settling the question of the effect of pregnancy on carbohydrate metabolism in the shorter medium term.An integrated approach to the study of carbohydrate metabolism in pregnancy would be of scientific and clinical relevance. Quantification of medium-long term carbohydrate metabolism status has been attempted using glycated haemoglobin (GHb).
While some reports shows a steady decline GHb levels in pregnancy, others have reported an increase (9). Other indicators of medium glycaemia in pregnancy might therefore be desirable.

Glycated plasma protein (GPP) or also referred to as fructosamine may better reflect overall trimester glycaemic changes than GHb levels. GPP would reflect the lifespan of plasma proteins while $\mathrm{GHb}$ reflects the life span of the red cells (9-10). The purpose of this cross-sectional study was therefore to determine the effect of pregnancy on medium carbohydrate metabolism using the levels of GPP and to assess whether medium carbohydrate metabolism in the African improves or deteriorates in pregnancy.

\section{SUBJECTS, MATERIALS AND METHODS}

One hundred and eighteen women at various stages of gestation were recruited from the ante- natal clinic of our hospital. Thirty-two non pregnant and non-diabetic women were also recruited as controls in addition to another group of 32 nonpregnant but diabetic women. Subjects with increased risk of DM were excluded from the controls and pregnant women. The study was approved by the Ethics and Research Committee of the Hospital. Venous blood samples were drawn from consenting subjects in the fasting state into heparinised bottles and fluoride oxide bottles for plasma proteins and sugar respectively. Plasma glucose was determined by the glucose oxidase method. Levels of glycated plasma proteins were determined by the thiobarbituric acid (TBA) method of Fluckinger and Winterhaeter as modified by McFarlan et al, (11-13). For each 
sample total plasma protein (TPP) was also determined using standard Biuret technique (14). The heparinised blood was centrifuged to separate the plasma from the solid elements of the blood. For GPP, the plasma was dialysed overnight to remove free glucose. The plasma was then stored at $-20^{\circ} \mathrm{C}$ until assay of GPP was performed. The plasma samples were incubated at $100^{\circ} \mathrm{C}$ for five hours. A fructose standard was run in each assay. Results of GPP are expressed as fructose equivalents and corrected for TPP as follows

GPPc $=$ Observed GPP $(\mathrm{mmol} / 1$ fructose $) /($ TPP in $\mathrm{g} / \mathrm{l})$ where GPPc is GPP reading corrected for TPP. Statistically GPP and FPG levels are presented as mean (SD). Significance of differences between groups was determined by student's $t$ test. The relationship between parametric values was determined using Pearson's product moment correlation coefficient. The level of statistical significance of differences or correlation coefficient is set at $p=0.05$.

\section{RESU LTS}

The characteristics of the study participants are shown in table 1

Tabel 1: Characteristics of Study Participants

\begin{tabular}{llll}
\hline Subjects & Subtotal & Age years & $\begin{array}{l}\text { Gestational } \\
\text { age (week) }\end{array}$ \\
\hline $\begin{array}{l}\text { First trimester } \\
\begin{array}{l}\text { Second trimester } \\
\text { Third trimester }\end{array}\end{array}$ & 46 & $27.1(4.6)$ & $10.1(2.13)$ \\
$\begin{array}{l}\text { Non-pregnant } \\
\text { Women }\end{array}$ & 32 & $28.4(4.2)$ & $17.60(3.40)$ \\
$\begin{array}{l}\text { Diabetic non } \\
\text { pregnant }\end{array}$ & 32 & $28.8(5.7)$ & $33.7(4.70)$ \\
\hline
\end{tabular}

Age is chronological and in years while gestational age is in weeks. The mean ages were not significantly different between the trimesters but the diabetic group was much older than the other groups $(p<0.01)$.

\section{GPPAssay Performance}

The intra-assay and inter-assay coefficients of Variation percent (CV \%) using a sample from a non-diabetic serum were $4.4 \%$ and $6.6 \%$ respectively with mean sample values of $37(1.7) \mathrm{mmol} / \mathrm{l}$ and $37.4(2.5) \mathrm{mmol} / \mathrm{l}$. For a diabetic serum with mean values of $48.8(2.9 \mathrm{mmol} / \mathrm{l}$ and $49.3(4.0) \mathrm{mmol} / \mathrm{l}$, the $\mathrm{CV} \%$ were respectively $5.9 \%$ and $8.1 \%$. The values of the $\mathrm{CV} \%$ were all within accepted ranges for this type of assay.

\section{Fasting Plasma Glucose}

The fasting plasma glucose (FPG) levels of the various strictly groups are shown in table: 2

\section{Table 2: Fasting Plasma Glucose Value in Groups of Participants}

Study group Mean (SD) FPG mg/dl

First trimester pregnancy

Second trimester pregnancy

Third trimester pregnancy

Non-pregnant non-diabetic

Non-pregnant but diabetic
$61.5(11.1)$

$62.3(14.9)$

59.1(11.9)

$78.2(20.2)$

248.7(78.1)
Between the trimesters the FPG values were not significantly different from one another and no clear trend was discernible in the pattern of the FPG in the pregnant groups. However the FPG levels in pregnancy were significantly lower than the mean for the non-pregnant women $(p<0.01)$ and more so than the diabetic women $(p<0.001)$.

\section{Glycated Plasma Proteins}

Table 3 is a comparison of the mean values of the GPP of the pregnant and non-pregnant groups.

Table 3: Glycated plasma protein values in pregnant and non-pregnant women

\begin{tabular}{ll}
\hline Study group & $\begin{array}{l}\text { Mean (SD) GPP (mmol/ } \\
\text { 1 fructose) equivalent }\end{array}$ \\
\hline First trimester & $33.4(4.9)$ \\
Second trimester & $34.7(5.5)$ \\
Third trimester & $35.9(5.8)$ \\
Non-pregnant Non-DM & $35.1(4.8)$ \\
Non-pregnant but diabetic & $58.5(6.1)$ \\
\hline
\end{tabular}

*Significantly higher than other trimesters and non-pregnant non diabetic group

The diabetic group had the highest mean GPP values, significantly higher than all other groups. Of the pregnant nondiabetic groups, GPP values progressively increased from the first trimester to peak in the third trimester. GPP values in the first and second trimesters were insignificantly lower than the mean for the non-pregnant non-diabetic group. The GPP value in the third trimester was significantly higher than those of the earlier trimesters and that in the non-pregnant women $(p<0.05)$.

\section{Relationship Between Fasting Plasma Glucose and Glycated Plasma Proteins}

Table 4 shows the relationship between FPG and GPP in all study groups. The correlation coefficient between FPG and GPP values were low and insignificant in the first two trimesters. However in the third trimester and in the diabetic non pregnant the coefficients were high and significant.

\section{Table 4: Relationship between FPG and GPP}

\begin{tabular}{ll} 
Study group & $\begin{array}{l}\text { Correlation coefficient } \\
\text { of FPG v GPP (r) }\end{array}$ \\
\hline
\end{tabular}

\begin{tabular}{ll}
\hline First trimester (26) & $+0.350^{*}$ \\
Second trimester (45) & $+0.237^{*}$ \\
Third trimester (47) & $+0.467^{*}$ \\
Non-pregnant women (32) & $+0.486^{*}$ \\
Non-pregnant but DM & $+0.766^{*}$
\end{tabular}

*Significantly higher than other trimesters and non-pregnant non diabetic group

\section{DISCUSSION}

Our GPP results in pregnancy are in keeping with the traditional view that carbohydrate tolerance worsens in pregnancy especially in late pregnancy, this contrary to some earlier reports. Reproducibility of plasma glucose in OGTT may be difficult. The usage of the glycated proteins should obviate 
the need for depending on repeated OGTTs in these subjects (1). There has also been a tendency towards contradicting results of carbohydrate metabolism in pregnancy using GPP and GHb (9). While $\mathrm{GHb}$ values have been shown to decrease initially and then rise in late pregnancy, GPP levels were reported to exhibit a progressive fall in pregnancy. The disparity between results of $\mathrm{GHb}$ and GPP charges in pregnancy appear to have been a failure to correct for TPP concentration, which fell progressively with advancing pregnancy. When correction is made for such a fall, as we have done in this work, the apparent fall is lost. Instead, peaking of GPP levels was noticed with advancing pregnancy. This increase was most marked in the third trimester when values did not only exceed those of the preceding trimester but also were higher than those of the non-pregnant non-diabetic women. It would seem that in the African woman, carbohydrate metabolism first improves and then deteriorates progressively with advancing gestational age. This pattern contrasts with some earlier reports from the continent which showed either no change or showed an improvement in carbohydrate tolerance status. Our urban subjects might not have been comparable with rural subjects studied by Swai et al., (5). Glucose absorption in pregnancy is variable and thus results of OGTT may be difficult to reproduce. While Lutale at al, (7) concluded that carbohydrate tolerance shows little change in urban Eastern African women, their mean post glucose load plasma glucose values did show a decrease at one hour and two hours in post partum. This might suggest that response glucose load by their subjects might have been relatively impaired in pregnancy. Our studies did not extend to the post partum period to enable us make appropriate comparison.

We conclude that FPG in pregnancy including late pregnancy in the African women is lower than in the non-pregnant state and appears to decrease further with advancing gestational age. On the other hand medium term carbohydrate tolerance using levels of GPP show progressive impairment of carbohydrate tolerance with advancing pregnancy. FPG and GPP may reflect different aspects of carbohydrate metabolism in pregnancy. The insulin resistance and carbohydrate intolerance in pregnancy may be better captured by GPP. We did not study the relationship between GPP and OGTT results. It would be of interest to compare such results. Finally, the GPP result reinforces the accepted view of the lack of usefulness in making diagnosis of carbohydrate intolerance in pregnancy from FPG. What role GPP can play in diagnosis and monitoring of carbohydrate intolerance in pregnancy will need to be further elucidated.

\section{ACKNOWLEDGEMENT}

Our appreciation to Dohwodese Ohwovoriole for his word processing assistance.

\section{REFERENCES}

1. Kuhl C. Glucose metabolism during and after pregnancy in normal and gestational diabetic women. Acta Endocrinol, 1975; 79:709-719.

2. Lind T, Billewicz WZ, Brown G. A serial study of changes in OGTT's during pregnancy. J Obstet Gynecol, 1973; 80:1033-1039.

3. Jackson WPU. Is pregnancy diabetogenic? Lancet, 1969; 2: 1329.

4. Fraser RB. The effect of pregnancy on normal range of the OGTT in Africans. East African Med J, 1981; 58:90-94.

5. Swai ABM, Kitange HM, McLarty DG, Kilima PM, Masuki G, Mtinangi BC, Albeati KGMM. No deterioration of OGTT during pregnancy in rural Tanzania. Diabetic Med, $1991 ; 8: 254-257$.

6. Famuyiwa OO, Amiohin RA, Adelunsi BO. OGTT in healthy pregnant Nigerian women. Diabetes Care, 1988; 11:412-415.

7. Lutale JK, Justesem A, Swai ABM, Alberti KGMM, McLarty DF. Glucose tolerance during and after pregnancy in non-diabetic women in an urban population in Tanzania. Diabetes Care, 1993:16: 575-577.

8. Oladunni G,TaylorJA, Mudie HA,Adeshima O,Akande EV. OGTT and insulin levels in pregnant and post partum women. Trop J Obstet Gynaecol, 1981 ;2:39-46.

9. Morris AM, ArnoldS, Grand S, Jean L, Hon C. Longitudinal assessment of glycosylated blood protein concentration in normal pregnancy ad gestational diabetes. Diabetes Care, 1986; 9: 107-109.

10. OhwovorioleAE, Kuti JA, Johnson TO. Influence of methodology on glycosylated haemoglobin values in Nigerian subjects with sickle cell haemoglobin party. Annals Clinical Lab Science, 1884; 14: 265-269.

11. FluckingerR, WinterhalterKH. In vitroSynthesisofHbAlc. FEBSLETT, 1976:71:356-360.

12. Kennedy L, McCause DR, Coulter D et al. Effect of minor fluctuation in albumin levels on serum fructosamine. Diabetes Care, 1986; 9:107-109.

13. McFairtand KF, Catland FW, Day JF et al. Non-enzymatic glycosylation of serum protein in diabetes mellitus. Diabetes, 1979; 28:1071-1074.

14. Gronali AG, Total plasma proteins estimation by Biuret method. JBiol Chem, 1949; 177: 557- 566. 